\title{
Infrared thermo-spectroscopic imaging of prostate cancer tissue
}

\author{
by Cai Li Song*, Meguya Ryu**, Junko Morikawa**, Archana Kothari ${ }^{\star * *}$, Sergei G. Kazarian* \\ *Department of Chemical Engineering, Imperial College London, South Kensington Campus, London \\ SW7 2AZ, United Kingdom. \\ ** Department of Materials Science and Engineering, Tokyo Institute of Technology, Ookayama, Meguro, \\ Tokyo 152-8552, Japan. \\ *** Department of Pathology, Kingston Hospital, KT2 7QE, Surrey, United Kingdom.
}

\begin{abstract}
:
A system that combines dispersive infrared micro-spectroscopic imaging and thermography has been developed to study the effect of thermal radiation on the infrared absorption spectra of prostate biopsy samples. The system allows the distribution of thermal signal intensity as a function of emissivity to be interpreted from the integrated absorbance obtained by spectroscopic imaging. Biochemical differences between cancer and benign areas within the specimens are identified in the spectra. Side-by-side comparison of H\&E stained adjacent tissue sections with infrared images constructed before and after removal of thermal effect showed that the latter strongly support differentiation of regions within tissues. The use of spectral bands at discrete wavelengths significantly reduced spectral acquisition time, making this technique promising as a future clinical diagnostic tool. A systemic methodology was implemented to process the data, firstly by k-means clustering on the second derivative spectra without a priori knowledge, followed by PCA analysis. Four distinct regions within the tissue samples were successfully classified based on the antisymmetric stretching mode of methylene functional group. Separation between data in clusters occurs when projecting spectra on a PCA score plot on a plane made by first two PCs. The significance of the disparity was verified with statistical test.
\end{abstract}

\section{Introduction}

Cancer diagnosis performed by experienced pathologists, which relies on visual inspection of morphological changes in the tissue through staining, has been the gold standard for establishing the presence of malignant tumours and classifying them. However there have been numerous cases where observations by pathologists led to falsely predicted behaviour characteristic of malignant disease or benign lesions. Infrared spectroscopic imaging has numerous advantages over the conventional histopathology method. Infrared (IR) micro-spectroscopy offers high chemical specificity, thereby can be used as a label-free powerful method for disease diagnosis. In brief, it is a vibrational spectroscopic technique such that molecular vibrations of a functional group absorb at their characteristic wavenumbers. The suitability of IR spectroscopic technique for the examination of biological samples to objectively characterise the present components and their distribution has been shown in previous studies. [1] It has extensively been used to observe prostate, cervix, breast, colon, thyroid, and skin cancer.

\section{Methods and materials}

In this study, a system that combines dispersive infrared micro-spectroscopic imaging and thermography has been developed to study the effect of thermal radiation on the infrared absorption spectra of healthy and cancer prostate tissues. Prostate biopsy specimens, microtomed at $3 \mu \mathrm{m}$ thickness from paraffin-embedded specimen block, were obtained from Kingston Hospital (London, UK). For each sample, serial sections were taken, one of which was mounted onto a $\mathrm{CaF}_{2}$ substrate with the adjacent section mounted onto a glass slide and stained with haematoxylin and eosin (H\&E) by pathologists. A Gleason score of $3 \times 3$ was assigned to the areas of malignancy identified within the H\&E stained tissues. The sections deposited on $\mathrm{CaF}_{2}$ window were de-paraffinized prior to IR measurement. The setup of the thermo-spectroscopic system is shown in Fig. 1. [2] A mechanical chopper is used for alternate acquisition of IR and thermal images by cutting off the IR light. An FPA of $128 \times 128$ pixels was used to capture the IR signal. The pixel size is $30 \mu \mathrm{m} \times 30 \mu \mathrm{m}$, giving a total field of view of $512 \mu \mathrm{m} \times 512 \mu \mathrm{m}$ under 7.5 $\times$ magnifications.

\section{Results and discussions}

Analyses on the IR chemical images before and after removal of thermal emission contribution revealed that the thermal effect is a significant contribution which needs to be removed prior to further data processing with unsupervised machine learning technique such as k-means clustering and principal component analysis (PCA). [3] We have also successfully reduced the acquisition time by using discrete wavelengths for spectroscopic imaging measurements and showed that the chemical images obtained have comparable spatial resolution to that obtained from FTIR spectroscopic imaging. Biochemical information regarding concentration of fatty acids in the tissue has been obtained using spectral bands within a spectral range of $3000-2800 \mathrm{~cm}^{-1}$. In particular, we concluded that spectral band of antisymmetric stretching of methylene group at $2921 \mathrm{~cm}^{-1}$ is a sensitive biomarker for differentiation of cancerous regions from surrounding stroma of prostate biopsy tissues. The false color map 
generated from k-means clustering reflects the distribution of the lipid composition changes in prostate cancer tissues, which was well related to the morphological changes in the cancerous regions observed from adjacent H\&E stained sections. Based on these findings and analyses, the demonstrated experimental set-up and procedures show promise as a label-free method in studying biological specimens after removal of effect originating from thermal radiation reaching the detector. Furthermore, the ratios of the absorbance of the spectral bands corresponding to the stretching modes of $\mathrm{CH}_{2}$ and $\mathrm{CH}_{3}$ functional groups show potential to be biomarkers for prostate cancer diagnosis. The outcome of this study is useful for establishing a reliable framework for future studies on prostate cancer diagnosis (Fig. 2).

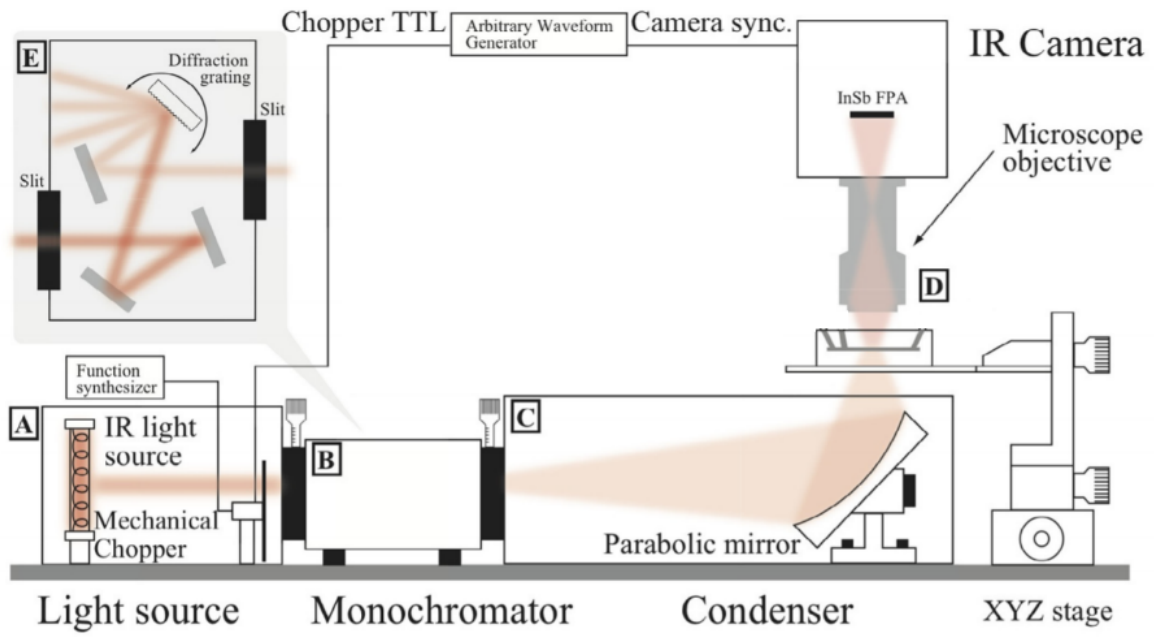

Fig. 1: Schematic view of the setup for the IR thermo-spectroscopic system (adapted from Ryu et al. [2])
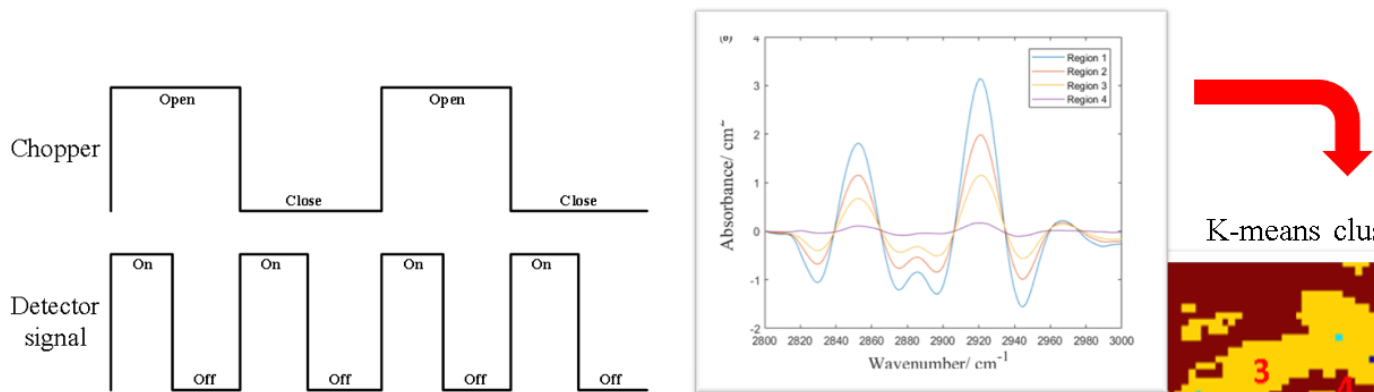

K-means clustering
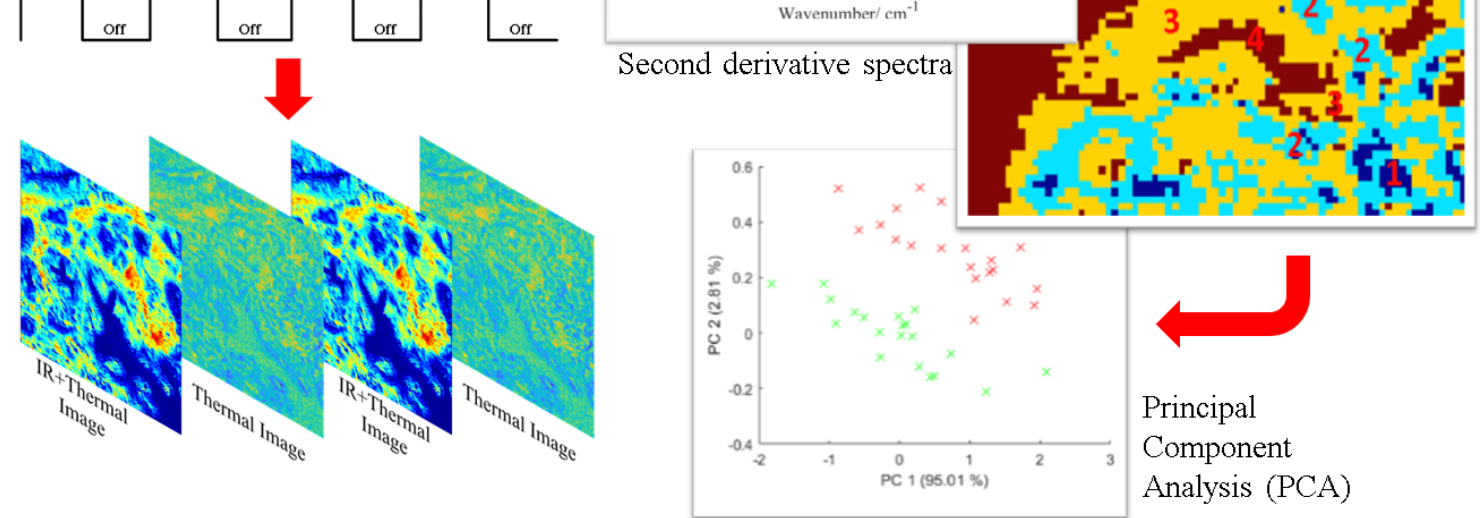

Fig. 2: The workflow of data processing and the results obtained (adapted from Song et al. [3])

\section{REFERENCES}

[1] M. J. Baker, H. J. Byrne, J. Chalmers, P. Gardner, R. Goodacre, A. Henderson, S. G. Kazarian, F. L. Martin, J. Moger, N. Stone, J. Sule-Suso. The Analyst. 2018, 143, 1735-1757.

[2] M. Ryu, J. A. Kimber, T. Sato, R. Nakatani, T. Hayakawa, M. Romano, C. Pradere, A. A. Hovhannisyan, S. G. Kazarian, J. Morikawa. Chemical Engineering Journal. 2017, 324, 259-265.

[3] C. Song, M. Ryu, J. Morikawa, A. Kothari and S. Kazarian. Journal of Biophotonics. 2018, 11, p. e201800187. 